\title{
Huffman Coding with Non-Sorted Frequencies
}

\author{
Shmuel T. Klein and Dana Shapira
}

\begin{abstract}
A standard way of implementing Huffman's optimal code construction algorithm is by using a sorted sequence of frequencies. Several aspects of the algorithm are investigated as to the consequences of relaxing the requirement of keeping the frequencies in order. Using only partial order may speed up the code construction, which is important in some applications, at the cost of increasing the size of the encoded file.
\end{abstract}

\section{Introduction}

Huffman's algorithm [8] is one of the major milestones of data compression, and even though more than half a century has passed since its invention, the algorithm or its variants find their way into many compression applications to this very day. The algorithm repeatedly combines the two smallest frequencies, and thus stores the set of frequencies either in a heap or in sorted form, yielding an $\Omega(n \log n)$ algorithm for the construction of the Huffman code, where $n$ is the size of the alphabet to be encoded.

Conditions under which Huffman's algorithm yields some generalized optimality properties have been studied in the past, see, e.g. [7, 12]. It is well known that there is no uniqueness in two quite different senses: a given probability distribution might yield more than a single (optimal) Huffman tree, and different distributions may on the other hand correspond to the same tree, see $[11,1]$. Working with a sorted set of frequencies is indeed a sufficient condition to get an optimal code, but the condition is not necessary. In certain cases, one can get optimal results even if the frequencies are not fully sorted, in other cases the code might not be optimal, but very closely so. On the other hand, relaxing the requirement of keeping the frequencies in order may yield time savings, as the generation

*This paper has been presented at the Prague Stringology Club, PSC-2008, Prague, Czech Republic, (2008). 
of the code, if the frequencies are already given in order, or if their order can be ignored, takes only $O(n)$ steps.

One might object that since the alphabet size $n$ can often be considered as constant relative to the size of the text to be encoded, there is no much sense in trying to improve the code construction process, and any gained savings will only marginally affect the overall compression time. But there are other scenarios for which the above mentioned effort may be justifiable: the ratio between the sizes of the text and the code is not always very large; instead of using a single Huffman code, better results are obtained when several such codes are used. For example, when the text is considered as being generated by a first order Markov process, one might use a different code for the successors of the different characters. When dynamic coding is used, the code is rebuilt periodically, sometimes even after each character read.

The loss incurred by not using an optimal (Huffman) code is often tolerable, and other non-optimal variants with desirable features, such as faster processing and simplicity have been suggested, for example Tagged Huffman codes [5], EndTagged Dense codes [3] and $(s, c)$-Dense codes [2]. Similarly, the loss of optimality caused by moving to not fully sorted frequencies can also be acceptable in certain applications, for example when based on estimations rather than on actual counts. In a dynamic encoding of a sequence of text blocks $B_{1}, B_{2}, \ldots$, block $B_{t}$ is often encoded on the basis of the character frequencies in $B_{1}, \ldots, B_{t-1}$. The encoder could use the frequencies from block $B_{t}$ itself, but deliberately ignores them because they are yet unknown to the decoder. By using the frequencies gathered up to block $B_{t-1}$ only, decoding is possible without transmitting the code itself. The accuracy, however, of these estimates is based on the assumption that block $t$ is similar to the preceding ones as to the distribution of its characters. If this assumption does not hold, the code may be non-optimal anyway, so an additional effort of producing an optimal code for a set of underlying frequencies that are not reliable, may be an overkill.

In the next section, we investigate some properties of the Huffman process on non-sorted frequencies. Section 3 then deals with a particular application, designing an algorithm for the dynamic compression of a sequence of data packets, and report on some experiments. In Section 4 we investigate whether a similar approach may have applications to other compression schemes than Huffman's.

\section{Using non-sorted frequencies}

The following example shows that working with sorted frequencies is not a necessary condition for obtaining optimality. Consider the sequence of weights $\{7,5,3,3$, $2,2\}$, yielding the Huffman tree in Figure 1a. If we start with a slightly perturbed sequence $\{7,5,3,2,3,2\}$ and continue according to Huffman's algorithm, we get the tree in Figure 1b, which is still optimal since its leaves are on the same levels as before, but it is not a Huffman tree, in which we would not combine 2 with 
3. The tree of Figure 1c corresponds to starting with the sorted sequence, but not keeping the order afterwards, working with the sequence $\{7,5,6,4\}$ instead of $\{7,6,5,4\}$ after two merges.

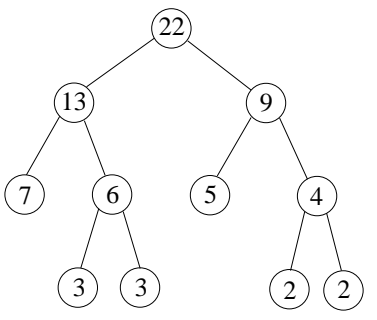

(a)

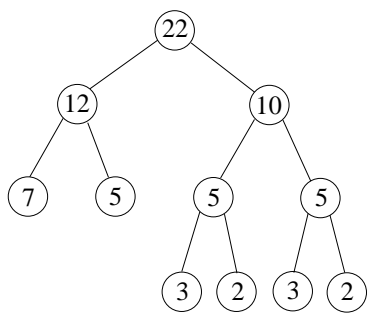

(b)

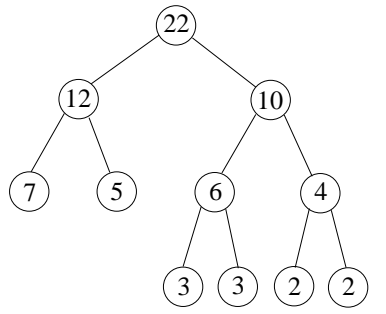

(c)

Figure 1: Optimal trees

Obviously, not paying at all attention to the order of the weights can yield very bad encodings. Consider a typical sequence of weights yielding a maximally skewed tree, that is, a tree with one leaf on each level (except the lowest level, on which there are two leaves). The Fibonacci sequence is known to be the one with the slowest increasing pace among the sequences giving such a biased tree [9], but for the ease of description we shall consider the sequence of powers of 2 , more precisely, the weights $1,1,2,4, \ldots, 2^{n}$, for some $n$.

Applying regular Huffman coding to this sorted sequence, we get

$$
S_{\mathrm{Huf}}=(n+1)+\sum_{i=0}^{n}(n-i+1) 2^{i}=2^{n+2}-2
$$

as total size of the encoded file. If one uses the same skewed tree, but assigns the codewords in reverse order, which can happen if the initial sequence is not sorted and the tree is built without any comparisons between weights, the size of the encoded file will be

$$
S_{\mathrm{rev}}=1+\sum_{i=0}^{n}(i+2) 2^{i}-2^{n}=(n+1) 2^{n+1}-2^{n}+1 .
$$

The ratio $S_{\text {rev }} / S_{\text {Huf }}$ may thus increase linearly with $n$, the size of the alphabet.

We therefore turn to a more realistic scenario, in which some partial ordering is allowed, but requiring an upper bound of $O(n)$ order operations, as opposed to $\theta(n \log n)$ for a full sort. Indeed, the simplest implementation of Huffman coding, after an initial sort of the weights, is keeping a sorted linked list, and repeatedly removing the two smallest elements and inserting their sum in its proper position, overall a $\theta\left(n^{2}\right)$ process. Using two queues $Q_{1}$ and $Q_{2}$, the first for the initial weights and the other for those created by adding two previous weights, the complexity can be reduced to $O(n)$ because the elements to be inserted into $Q_{2}$ appear in order [13]. If one starts with a sequence which is inversely sorted, the first element to be inserted into $Q_{2}$ will be the largest; hence if one continues as in the original 
algorithm by extracting either the two smallest elements of $Q_{1}$, or those of $Q_{2}$, or the smallest from $Q_{1}$ and that of $Q_{2}$, the first element of $Q_{2}$ will be used again only after the queue $Q_{1}$ has been emptied. The resulting tree is thus a full binary tree, with all its leaves on the same level if $n$ is a power of 2 , or on two adjacent levels if not. The depth of this tree, for the case $n=2^{k}$, will be $k$. Returning to the above sequence of weights, the total size of the encoded file will thus be

$$
S_{\text {fixed }}=\log n\left(1+\sum_{i=0}^{n} 2^{i}\right)=2^{n+1} \log n .
$$

The ratio $S_{\text {fixed }} / S_{\text {Huf }}$ still tends to infinity, but increases only as $\log n$ as opposed to $n$ above.

One of the ways to get some useful partial ordering in linear time is the one used in Yao's Minimum Spanning tree algorithm [16]: a parameter $K$ is chosen, and the set of weights $W$ is partitioned into $K$ subsets of equal size $W_{1}, \ldots, W_{K}$, such that all the elements of $W_{i}$ are smaller than any element in $W_{i+1}$, for $i=$ $1, \ldots, K-1$, but without imposing any order within each of the sets $W_{i}$. The total time for such a partition is only $O(n \log K)$, using repeatedly an $O(n)$ algorithm for finding the median first of the whole set $W$, then of its two halves (the $n / 2$ lower and the $n / 2$ upper values), then of the quarters, etc. Starting with such a partition and continuing with the help of two queues, one gets an overall linear algorithm, since $K$ is fixed. On the other hand, $K$ can be used as a parameter of how close the initial ordering should be to a full sort.

\begin{tabular}{|l|cccc|}
\hline & 1-grams & 2-grams & 3-grams & 4-grams \\
\hline English & 52 & 808 & 6026 & 21886 \\
French & 131 & 2965 & 18864 & 56078 \\
\hline
\end{tabular}

TABLE 1: Alphabet sizes

To empirically test this partition approach, we chose the following input files of different sizes and languages: the Bible (King James version) in English, and the French version of the European Union's JOC corpus, a collection of pairs of questions and answers on various topics used in the ARCADE evaluation project [14]. To get also different alphabet sizes, the Bible text was stripped of all punctuation signs, whereas the French text has not been altered. We then also considered extended alphabets, consisting of bigrams, trigrams and 4-grams, that is, the text was split into a sequence of $k$-grams, $1 \leq k \leq 4$, and for fixed $k$, the set of the different non-overlapping $k$-grams was considered as an alphabet. Table 1 shows the sizes of the alphabets so obtained.

Each sequence of weights was then partitioned as explained above into $K$ equal parts, with $K=1,2,4,8, \ldots$, where in each part the original lexicographic order of the elements has been retained. Figure 2 plots the average number of bits 

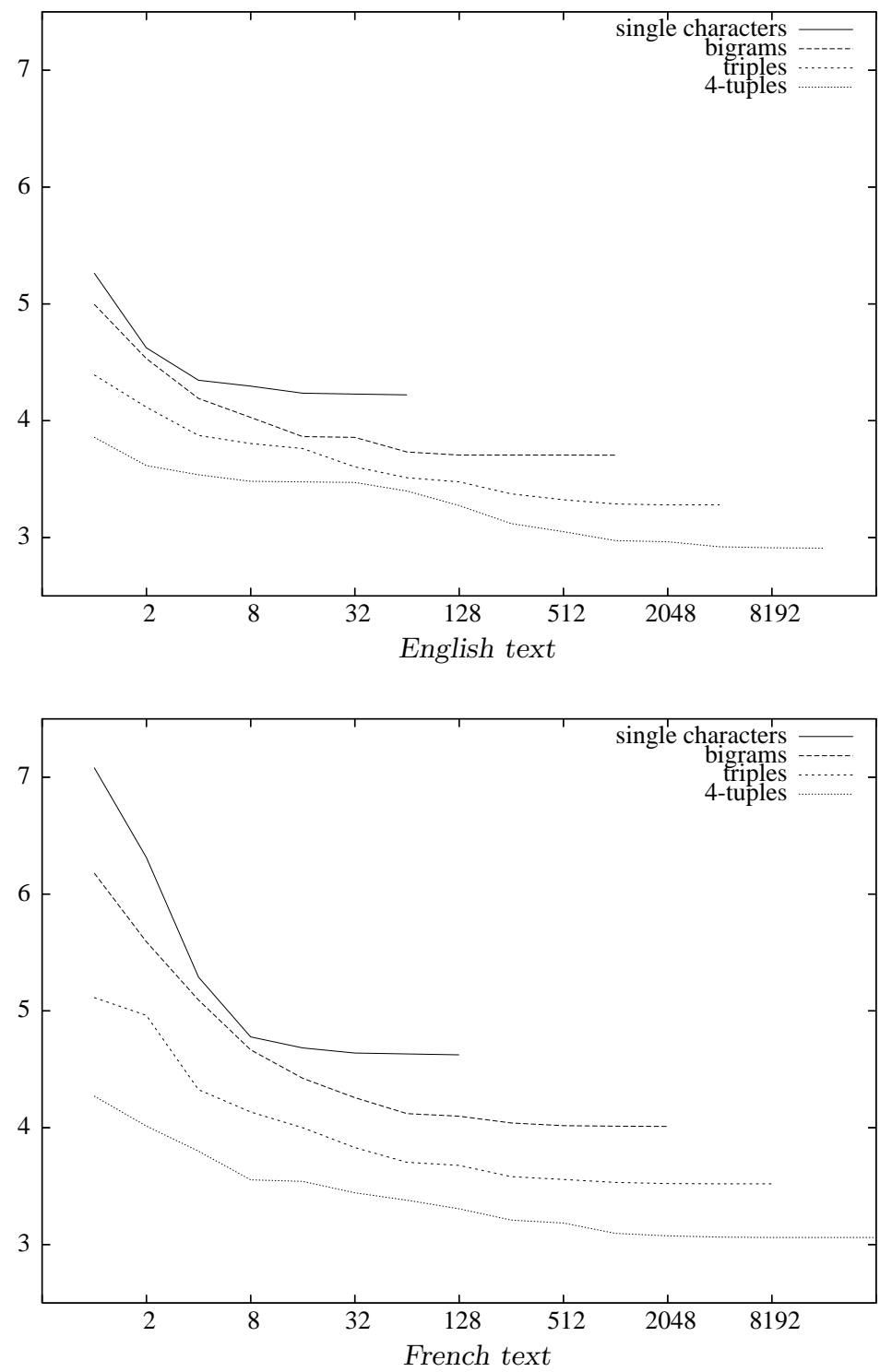

Figure 2: Average number of bits per char as function of number of blocks in partition

needed to encode a single character as function of the number of partition parts $K$. All the plots exhibit a decreasing trend and obviously converge to the optimum when $K$ reaches the alphabet size, but it should be noted that the convergence pace is quite fast. For example, for the 4 -tuple alphabets, using $K=1024$ corresponding 
to 10 partition phases, there is a loss of only $1.1 \%$ for the English and $2.2 \%$ for the French texts over the optimal Huffman code.

Another kind of partial ordering relates to a dynamic environment where the Huffman trees to be used are constantly updated. An application of this idea to a packet transmission system is discussed in the next section.

\section{Dynamic compression of a sequence of data packets}

Consider a stream of data packets $P_{1}, P_{2}, \ldots$ of varying sizes, which should be transmitted in compressed form over some channel. In practice, the sizes have great variability, ranging from small packets of several bytes up to large ones, spanning Megabytes. Compression of packet $P_{t}$ will be based on $P_{t-k}, P_{t-k+1}, \ldots, P_{t-1}$, where $k$ could be chosen as $t-1$ if one wishes to use the full history, or as some constant if the compression of each packet should only depend on the distribution in some fixed number of preceding packets.

Normally, after having processed $P_{t}$, the distribution of the weights should be updated and a new Huffman tree should be built accordingly. The weights of elements which did not appear earlier are treated similarly to the appearance of new elements in dynamic Huffman coding. We suggest, however, to base the Huffman tree reconstruction not on a full sort of the updated frequencies, but on a partial one obtained from a single scan of a bubble-sort procedure. For the formal description, let $s_{i}, 1 \leq i \leq n$, be the elements to be encoded. These elements can typically be characters, but could also be pairs or triplets of characters as in the example above, or even words, or more generally, any set of strings or more general elements, as long as there is some unambiguous way to partition the text into a sequence of such elements. Let $f\left(s_{i}\right)$ be the frequency of $s_{i}$ and note that we do not require the sequence $f\left(s_{1}\right), f\left(s_{2}\right), \ldots$ to be non-decreasing. The update algorithm to be applied after each block is:

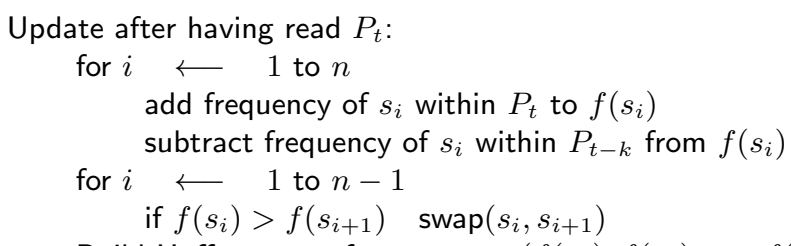

Build Huffman tree for sequence $\left(f\left(s_{1}\right), f\left(s_{2}\right), \ldots, f\left(s_{n}\right)\right)$ using two queues

The gain of using only a single iteration of possible swaps is not only in processing time. It also allows a more moderate adaptation to changing character distributions in the case of the appearance of some very untypical data packets. Only if the changed frequencies persist also in several subsequent packets, will the Huffman tree gradually change its form to reflect the new distributions. On the other hand, if the packets are homogeneous, the procedure will zoom in on the optimal order after a small number of steps. 
To simulate the above packet transmission algorithm, we took the English and French texts mentioned earlier, and partitioned them into sequences of blocks, each representing a packet. For simplicity, the block size has been kept fixed. The tests were run with single character and bigram alphabets. The following methods were compared:

1. Blocked - Block encoding: each block uses the Huffman tree built for the cumulative frequencies of all the preceding blocks to encode its characters.

2. Bubble - Using one bubble-sort iteration: each block uses the cumulative frequencies of all previous blocks as before, but after each block, only a single bubble-sort iteration is performed on the frequencies instead of sorting them completely. Huffman's algorithm is then applied on the non-sorted sequence of weights.

3. Bubble-For- $k$ - Forgetful variant of Bubble: each block uses the cumulative frequencies not of all, but only the $k$ previous blocks $(k \geq 0)$. The frequencies of blocks that appear more than $k$ blocks earlier are thus not counted for building the Huffman tree of the current block. This allows a better adaptation in case of heterogeneous blocks, at the price of slower convergence in the case of a more uniform behavior of the character distributions within the blocks.

For the last case we considered both Bub-For-1 and Bub-For-5, using the frequencies of the preceding block only and of the last five blocks, respectively. The first block was encoded with a fixed length code using the full single character or bigram alphabet. After each block read, the statistics were updated and a new code was generated according to the methods above. The recorded time is that of the average code construction time per block, not including the actual encoding of the block.

\begin{tabular}{|cc|c|cccc|}
\hline \multirow{2}{*}{ Single characters } & $\begin{array}{c}\text { Block } \\
\text { size }\end{array}$ & Blocked & Bubble & $\begin{array}{c}\text { Bubble } \\
\text { For-1 }\end{array}$ & $\begin{array}{c}\text { Bubble } \\
\text { For-5 }\end{array}$ \\
\hline \multirow{4}{*}{ English } & \multirow{3}{*}{ Compression } & 200 & 4.112 & 5.532 & 5.697 & 5.607 \\
& & 2000 & 4.114 & 5.532 & 5.553 & 5.541 \\
& \multirow{3}{*}{ Time } & 10000 & 4.123 & 5.533 & 5.536 & 5.533 \\
\cline { 3 - 7 } & & 200 & 0.13 & 0.06 & 0.06 & 0.06 \\
& & 10000 & 0.63 & 0.44 & 0.27 & 0.27 \\
& \multirow{3}{*}{ Compression } & 200 & 4.699 & 1.32 & 1.13 & 1.26 \\
\cline { 3 - 7 } & & 2000 & 4.700 & 6.020 & 5.901 & 5.875 \\
& & 10000 & 4.705 & 6.022 & 5.834 & 5.825 \\
& \multirow{3}{*}{ Time } & 200 & 0.27 & 0.09 & 0.09 & 0.11 \\
& & 2000 & 0.49 & 0.30 & 0.30 & 0.31 \\
& & 10000 & 1.47 & 1.26 & 1.28 & 1.28 \\
\hline
\end{tabular}

TABLE 2: Dynamic compression of data packets using single characters 


\begin{tabular}{|cc|c|cccc|}
\hline \multirow{2}{*}{ Bigrams } & $\begin{array}{c}\text { Block } \\
\text { size }\end{array}$ & Blocked & Bubble & $\begin{array}{c}\text { Bubble } \\
\text { For-1 }\end{array}$ & $\begin{array}{c}\text { Bubble } \\
\text { For-5 }\end{array}$ \\
\hline \multirow{4}{*}{ English } & \multirow{3}{*}{ Compression } & 2000 & 3.805 & 5.061 & 5.061 & 5.061 \\
& & 10000 & 3.805 & 5.061 & 5.061 & 5.062 \\
& \multirow{3}{*}{ Time } & 20000 & 3.806 & 5.062 & 5.062 & 5.062 \\
\cline { 3 - 7 } & & 2000 & 30.1 & 7.3 & 9.0 & 11.6 \\
& & 10000 & 34.9 & 9.2 & 10.8 & 13.4 \\
& \multirow{3}{*}{ French } & 20000 & 37.4 & 11.1 & 12.9 & 15.2 \\
\cline { 3 - 7 } & \multirow{3}{*}{ Compression } & 2000 & 4.109 & 6.343 & 6.345 & 6.345 \\
& & 10000 & 4.109 & 6.342 & 6.344 & 6.344 \\
& & 20000 & 4.108 & 6.342 & 6.345 & 6.342 \\
\hline \multirow{3}{*}{ Time } & 2000 & 286.2 & 9.9 & 11.3 & 14.0 \\
& & 10000 & 286.6 & 11.1 & 12.9 & 16.1 \\
& & 20000 & 290.4 & 13.4 & 15.1 & 17.6 \\
\hline
\end{tabular}

TABLE 3: Dynamic compression of data packets using bigrams

Table 2 brings the results for the single character alphabets and Table 3 the corresponding values for the bigram alphabets. The block sizes used were 200, 2000 and 10000 for the single characters and 2000, 10000 and 20000 for the bigrams. The compression figures are given in bits per character and the time is measured in milliseconds.

As can be seen, there is a significant loss, on our data, in compression efficiency, when using non-sorted frequencies. The block size seems not to have an impact on the compression. For the bigrams, there is also no difference between the forgetful variants and that using all the preceding data blocks, but for the smaller single character alphabets, the compression using only the information of the few last blocks is marginally better on the French text, and worse on the English one. This can be explained by the different nature of the texts: The English Bible is one homogeneous entity, and its partition into blocks is purely artificial. We may thus expect that using more global statistics will yield better compression performance. The French text, on the other hand, consists of many independent queries and their answers, covering a very large variety of topics. Using the distribution of one block to compress a subsequent one may thus not always yield good results, so a variant which is able to "forget" a part of what it has seen, may be advantageous in this case.

The loss in compression is compensated by savings in sorting time. These savings are more pronounced for the larger bigram alphabets, but also noticeable for the character alphabets. The time is increasing with the size of the blocks, because a larger block gives more possibilities for a larger variability of the frequencies. The exception here is for the bigrams of the French text: the alphabet in this case is so large, that the block size has only a minor impact on the processing time. On the other hand, it is in this case that the savings using partial order are the most significant. 


\section{Relevance of partial sort to other compression schemes}

We check in this section whether the idea of not fully sorting the frequencies could be applicable to other compression methods.

\subsection{Arithmetic coding}

In fact, for both encoding and decoding using an arithmetic coder [15], the weights need not be in any specific order, as long as encoder and decoder agree upon the same. This has the advantage for the dynamic variant, that the same order of the elements can be used at each step, for example that induced by the lexicographic order of the elements to be encoded. Partial ordering is thus not relevant here.

\subsection{6-ary Huffman codes, $(s, c)$-dense codes, Fibonacci codes}

All these codes can be partitioned into blocks of several codewords having all the same length. For 256-ary Huffman, the codeword lengths are multiples of bytes, so that even for very large alphabets, it is very rare to get codewords longer than 3 or 4 bytes; the same is true for $(s, c)$-dense codes. It follows that, almost always, all the codewords can be partitioned into 3 or 4 groups, so a full sort is not even necessary. It suffices to partition the weights into these classes, as suggested above, just that the sizes of the blocks of the partition are not equal, but rather derived from the specific code.

For Fibonacci codes $[6,10]$, there are $F_{n}$ codewords of length $n+2$, where $F_{i}$ are Fibonacci numbers, and this set is fixed, just as for $(s, c)$-codes. The number of blocks here is larger, but even for an alphabet of one million characters, there are no more than 29 blocks, and the partition can be done in 5 iterations.

\subsection{Burrows-Wheeler Transform (BWT)}

At first sight, partially sorting seems to be relevant to BWT [4], as the method works on a string of length $n$ and applies all the $n$ cyclic rotations on it, yielding an $n \times n$ matrix which is then lexicographically sorted by rows. The first column of the sorted matrix is thus sorted, but BWT stores the last column of the matrix, which together with a pointer to the index of the original string in the matrix lets the file to be recovered. The last column is usually not sorted, but it often is very close to be sorted, which is why it is more compressible than the original string. The BWT uses a move-to-front strategy to exploit this nearly sorted nature of the string to be compressed.

One could think that since the last column is anyway only nearly sorted, then if the initial lexicographic sort of the matrix rows is only partially done, the whole damage would be that the last row will be even less sorted, so we would trade compression efficiency for time savings. However, the reversibility of BWT is based on the fact that the first column is sorted, so a partial sort would invalidate the whole method and not just reduce its performance. 


\section{Conclusion}

We have dealt with the simple idea of not fully sorting the weights used by Huffman's algorithm, expecting some time savings in applications where the sort is a significant part of the encoding process. This may include large alphabets, or using several alphabets like in dynamic applications, or when encoding according to a first order Markov chain. The tests showed that by using partial sorts, the execution time can be reduced at the cost of some loss in compression efficiency.

\section{References}

[1] Ahlswede, R.: Identification entropy. Information Transfer and Combinatorics, LNCS, 4123 2006, pp. 595-613.

[2] Brisaboa, N. R., Fariña, A., Navarro, G., and Esteller, M. F.: (s,c)-dense coding: an optimized compression code for natural language text databases. Proc. Symposium on String Processing and Information Retrieval SPIRE'03, LNCS, 2857 2003, pp. 122-136.

[3] Brisaboa, N. R., Iglesias, E. L, Navarro, G., and Paramá, J. R: An efficient compression code for text databases. Proc. European Conference on Information Retrieval ECIR'03, LNCS, 2633 2003, pp. 468-481.

[4] Burrows, M. And Wheeler, D. J.: A block-sorting lossless data compression algorithm. Technical Report SRC 124, Digital Systems Research Center, 1994.

[5] de Moura, E. S., Navarro, G., Ziviani, N., and Baeza-Yates, R.: Fast and flexible word searching on compressed text. ACM Trans. on Information Systems, 18 2000, pp. 113-139.

[6] Fraenkel, A.S. And Klein, S.T.: Robust universal complete codes for transmission and compression. Discrete Applied Mathematics, 64 1996, pp. 31-55.

[7] Glassey, C.R. And KarP, R.M.: On the optimality of Huffman trees. SIAM J. Applied Math., 31 1976, pp. 368-378.

[8] Huffman, D.: A method for the construction of minimum redundancy codes. Proc. of the IRE, 40 1952, pp. 1098-1101.

[9] Katona, G.H.O. And Nemetz, T.O.H.: Huffman codes and self-information. IEEE Trans. on Inf. Th., IT-11 1965, pp. 284-292.

[10] Klein, S.T. And Kopel Ben-Nissan, M.: Using Fibonacci compression codes as alternatives to dense codes. Proc. Data Compression Conference DCC-2008, 2008, pp. $472-481$.

[11] Longo, G. And Galasso, G.: An application of informational divergence to Huffman codes. IEEE Trans. on Inf. Th., IT-28 1982, pp. 36-43.

[12] PARKer JR., D.S.: Conditions for the optimality of the Huffman algorithm. SIAM J. on Computing, 9 1980, pp. 470-489.

[13] VAn LeEuwen, J.: On the construction of Huffman trees. Proc. $3^{\text {rd }}$ ICALP Conference, 1976, pp. 382-410.

[14] VÉronis, J. And LANGlais, P.: Evaluation of parallel text alignment systems: The ARCADE project. Parallel Text Processing, J. Véronis, ed., 2000, pp. 369-388. 
[15] Witten, I.H, Neal, R.M., And Cleary, J.G.: Arithmetic coding for data compression. Comm. of the ACM, 30 1987, pp. 520-540.

[16] YAO, A.C.C.: An $O(|E| \log \log |V|)$ algorithm for finding minimum spanning trees. Inf. Processing Letters, 4 1975, pp. 21-23.

Shmuel T. Klein

Department of Computer Science

Bar Ilan University

Ramat Gan 52900, Israel

e-mail: tomi@cs.biu.ac.il

Dana Shapira

Department of Computer Science

Ashkelon Academic College

Ashkelon 78211, Israel

e-mail: shapird@ash-college.ac.il 